\title{
On flows of viscoelastic fluids of Oldroyd type with wall slip
}

\author{
Christiaan Le Roux
}

\begin{abstract}
We consider the boundary-value problem for the steady isothermal flow of an incompressible viscoelastic liquid of Oldroyd type in a bounded domain with a Navier type slip boundary condition. We prove that under some restrictions on the material constants and the data, there exists a strong solution which is locally unique. The proof is based on a fixed point argument in which the boundary-value problem is decomposed into a transport equation and a Stokes system.
\end{abstract}

Mathematics Subject Classification (2010). Primary 76A10; Secondary 76D03.

Keywords. Viscoelastic fluid, Oldroyd fluid, slip boundary condition.

\section{Introduction}

The models of viscoelastic fluids formulated by Oldroyd [1, 2] (see [3, 4, 5] for alternative derivations and perspectives), especially the Oldroyd-B model, have been studied by several mathematicians since the pioneering work of Renardy [6] and Guillopé and Saut [7]-[10]. Many results regarding the well-posedness of boundaryvalue problems and initial-boundary-value problems for flows of liquids described by these and related non-Newtonian models have been established; see [11]-[46] and the references therein. These results deal with time-dependent or steady flows of compressible or incompressible fluids in bounded, unbounded, exterior or thin domains, in two or three dimensions, under various restrictions on the material constants and other data. A variety of numerical methods for the simulation of such flows have also been developed; see, e.g., [47]-[61].

Many of the numerical studies with Oldroyd type models focus on the stickslip problem that arises in the extrusion of polymer melts, where there is an abrupt transition from the no-slip boundary condition at the solid surface of the die to the shear-free slip boundary condition at the free surface of the extrudate and a resulting stress singularity. A similar stress singularity occurs at contact lines in free surface problems. In both problems, the stress singularity can be alleviated by applying a partial slip boundary condition (such as Navier's slip law) at the solid surface (see, e.g., $[62,63,64]$ ). Moreover, there has been an extensive experimental and theoretical investigation of the presence and role of wall slip (or apparent wall slip) in the onset of flow instabilities (spurt) and surface defects (melt fracture and 
sharkskin) in polymer extrusion processes (see [65, 66] and [67, Section 11.5.4]) and errors in experimental methods for molten polymers [67, Chapter 10]. These phenomena have much practical importance but are complex and only partly understood despite the large amount of work (see the references in $[65,66,67]$ ) devoted to the measurement, modeling and simulation of it.

On the other hand, the published well-posedness results for flows of Oldroyd type fluids, including those of [11]-[46], are concerned with flows in fixed domains and assume that there is no wall slip. To our knowledge, there is at present no well-posedness result for the die-swell free surface problem of an Oldroyd type fluid with partial slip at the solid surface. In fact, the much simpler problem of the flow in a fixed domain with partial wall slip has apparently not been addressed. The aim of the present article is to consider such a problem. For the sake of simplicity we consider flows with the Navier slip boundary condition, but the method of proof which is based on the decomposition of the problem into a transport equation for the extra-stress and a Stokes system for the velocity and pressure - can be adapted to non-linear slip boundary conditions such as those employed in [68]-[71], where the tangential traction is a non-monotone function of the slip velocity.

The outline of the paper is as follows. First we formulate the boundary-value problem (Section 1.1) and state our main result (Section 1.2). Then we define the notation (Section 2) and establish some auxiliary results (Section 3). Thereafter we prove Theorem 1 and indicate how it can be extended to problems with non-linear slip boundary conditions (Section 4). Lastly, we show that the solution is locally unique (Section 5).

\subsection{Problem formulation}

We consider a class of rate type models of homogeneous incompressible viscoelastic liquids, which we call Oldroyd type fluids. The Cauchy stress tensor is given by $\boldsymbol{T}=-\hat{p} \boldsymbol{I}+\boldsymbol{S}$, where $\hat{p}$ is the pressure and $\boldsymbol{S}$ is the extra-stress, which satisfies the equation

$$
\boldsymbol{S}+\lambda_{1} \frac{\mathcal{D}_{a}}{\mathcal{D} t} \boldsymbol{S}=2 \mu_{0}\left(\boldsymbol{D}+\lambda_{2} \frac{\mathcal{D}_{a}}{\mathcal{D} t} \boldsymbol{D}\right) \quad \text { in } \Omega .
$$

Here $\Omega$ is the flow domain and $\mathcal{D}_{a} / \mathcal{D} t$ denotes the objective time derivative

$$
\frac{\mathcal{D}_{a}}{\mathcal{D} t} \boldsymbol{S}=\frac{\partial \boldsymbol{S}}{\partial t}+\boldsymbol{v} \cdot \nabla \boldsymbol{S}+P_{a}(\nabla \boldsymbol{v}, \boldsymbol{S})
$$

where $a \in[-1,1]$ and

$$
\begin{aligned}
P_{a}(\nabla \boldsymbol{v}, \boldsymbol{S}): & =\boldsymbol{S} \boldsymbol{W}-\boldsymbol{W} \boldsymbol{S}-a(\boldsymbol{S} \boldsymbol{D}+\boldsymbol{D} \boldsymbol{S}) \\
& =\frac{1}{2}(1-a)\left(\boldsymbol{S} \nabla \boldsymbol{v}+(\nabla \boldsymbol{v})^{T} \boldsymbol{S}\right)-\frac{1}{2}(1+a)\left(\boldsymbol{S}(\nabla \boldsymbol{v})^{T}+(\nabla \boldsymbol{v}) \boldsymbol{S}\right)
\end{aligned}
$$

with $\boldsymbol{D}=\frac{1}{2}\left(\nabla \boldsymbol{v}+(\nabla \boldsymbol{v})^{T}\right)$ and $\boldsymbol{W}=\frac{1}{2}\left(\nabla \boldsymbol{v}-(\nabla \boldsymbol{v})^{T}\right)$. The material constants $\mu_{0}, \lambda_{1}$ and $\lambda_{2}$ are the viscosity coefficient, the stress relaxation time and the retardation time, respectively. We assume that

$$
0<\mu_{0}, \quad 0<\lambda_{2}<\lambda_{1} .
$$

The special case of the derivative (1.2) with $a=1(a=0, a=-1$, resp. $)$ is called the upper convected (corotational, lower convected, resp.) derivative and an Oldroyd type fluid with $a=1$ ( $a=-1$, resp.) is called an Oldroyd-B (Oldroyd-A, resp.) fluid. An Oldroyd-B fluid with $\lambda_{2}=0$ is known as a Maxwell fluid; with 
$0<\lambda_{2}<\lambda_{1}$ it is known as a Jeffreys fluid. In fact, equation (1.1) can be written as the Maxwell type equation

$$
\hat{\boldsymbol{E}}+\lambda_{1} \frac{\mathcal{D}_{a}}{\mathcal{D} t} \hat{\boldsymbol{E}}=2 \mu_{e} \boldsymbol{D} \quad \text { in } \Omega,
$$

where $\hat{\boldsymbol{E}}:=\boldsymbol{S}-2 \mu_{n} \boldsymbol{D}$ is the "elastic part" of the extra-stress, and $\mu_{n}:=\mu_{0} \lambda_{2} / \lambda_{1}$ and $\mu_{e}:=\mu_{0}\left(1-\lambda_{2} / \lambda_{1}\right)$ are the so-called Newtonian and elastic viscosity coefficients, respectively. With this notation, the conservation of linear momentum for a steady flow becomes

$$
\rho \boldsymbol{v} \cdot \nabla \boldsymbol{v}-\mu_{n} \Delta \boldsymbol{v}+\nabla \hat{p}=\operatorname{div} \hat{\boldsymbol{E}}+\rho \boldsymbol{f} \quad \text { in } \Omega,
$$

where $\rho$ is the density and $\boldsymbol{f}$ is the body force per unit mass. Furthermore, since the fluid is incompressible and the density is constant, the conservation of mass reduces to

$$
\operatorname{div} \boldsymbol{v}=0 \text { in } \Omega \text {. }
$$

We assume that the boundary, $\Gamma$, of $\Omega$ is impermeable. Thus

$$
\boldsymbol{v} \cdot \boldsymbol{n}=0 \quad \text { on } \Gamma
$$

where $\boldsymbol{n}$ denotes the outward unit normal vector. Moreover, we assume that the liquid-solid interaction is governed by Navier's slip condition,

$$
\left[\left(\hat{\boldsymbol{E}}+2 \mu_{n} \boldsymbol{D}\right) \boldsymbol{n}\right]_{\tau}+\hat{K}(\boldsymbol{v}-\boldsymbol{w})=0 \text { on } \Gamma,
$$

where $[\cdot]_{\tau}$ denotes the tangential component, $\hat{K}$ is a positive constant, $\boldsymbol{w}$ is the velocity of the solid surface and $\boldsymbol{w} \cdot \boldsymbol{n}=0$ on $\Gamma$. Thus, $\Omega$ is fixed but $\Gamma$ (or parts of $\Gamma$, which may be connected to each other or disconnected from other components of $\Gamma$ ) may undergo steady tangential motion, e.g., rotation or stretching. For this reason we do not assume that $\boldsymbol{w}$ is identically zero. This is also discussed in $[72$, Remark 1.2].

Lastly, define $\boldsymbol{E}=\rho^{-1} \hat{\boldsymbol{E}}, p=\rho^{-1} \hat{p}, \nu_{0}=\mu_{0} / \rho, \nu_{n}=\mu_{n} / \rho, \nu_{e}=\mu_{e} / \rho\left(\nu_{n}\right.$ and $\nu_{e}$ are the kinematic Newtonian and kinematic elastic viscosity coefficients, respectively), $K=\hat{K} / \rho$ and $\boldsymbol{A}=\nabla \boldsymbol{v}+(\nabla \boldsymbol{v})^{T}$. Then, from (1.6)-(1.10), we have the following boundary-value problem:

Problem 1. Find $(\boldsymbol{E}, \boldsymbol{v}, p)$ such that $\boldsymbol{E}$ is symmetric and

$$
\begin{aligned}
\boldsymbol{E}+\lambda_{1}\left(\boldsymbol{v} \cdot \nabla \boldsymbol{E}+P_{a}(\nabla \boldsymbol{v}, \boldsymbol{E})\right)-\nu_{e} \boldsymbol{A} & =0 & & \text { in } \Omega, \\
\boldsymbol{v} \cdot \nabla \boldsymbol{v}-\nu_{n} \Delta \boldsymbol{v}+\nabla p-\operatorname{div} \boldsymbol{E} & =\boldsymbol{f} & & \text { in } \Omega, \\
\operatorname{div} \boldsymbol{v} & =0 & & \text { in } \Omega, \\
{\left[\left(\boldsymbol{E}+\nu_{n} \boldsymbol{A}\right) \boldsymbol{n}\right]_{\tau}+K \boldsymbol{v} } & =K \boldsymbol{w} & & \text { on } \Gamma, \\
\boldsymbol{v} \cdot \boldsymbol{n} & =0 & & \text { on } \Gamma .
\end{aligned}
$$

To write Problem 1 in dimensionless form, choose a characteristic length $L$ and a characteristic speed $V$ and define $\boldsymbol{x}^{*}=L^{-1} \boldsymbol{x}$ for all $\boldsymbol{x} \in \bar{\Omega}, \Omega^{*}=\left\{\boldsymbol{x}^{*}: \boldsymbol{x} \in\right.$ $\Omega\}, \Gamma^{*}=\left\{\boldsymbol{x}^{*}: \boldsymbol{x} \in \Gamma\right\}, t^{*}=V L^{-1} t, \boldsymbol{E}^{*}\left(\boldsymbol{x}^{*}\right)=V^{-2} \boldsymbol{E}(\boldsymbol{x}), \boldsymbol{v}^{*}\left(\boldsymbol{x}^{*}\right)=V^{-1} \boldsymbol{v}(\boldsymbol{x})$, $\boldsymbol{w}^{*}\left(\boldsymbol{x}^{*}\right)=V^{-1} \boldsymbol{w}(\boldsymbol{x}), p^{*}\left(\boldsymbol{x}^{*}\right)=V^{-2} p(\boldsymbol{x}), \lambda_{1}^{*}=V L^{-1} \lambda_{1}, \lambda_{2}^{*}=V L^{-1} \lambda_{2}, \nu_{n}^{*}=$ $L^{-1} V^{-1} \nu_{n}, \nu_{e}^{*}=L^{-1} V^{-1} \nu_{e}, \boldsymbol{f}^{*}\left(\boldsymbol{x}^{*}\right)=L V^{-2} \boldsymbol{f}(\boldsymbol{x}), K^{*}=V^{-1} K$. Then, by writing equations (1.11)-(1.15) in terms of these dimensionless quantities and omitting the asterisks, we obtain a problem which is formally identical to Problem 1. Henceforth, we consider this dimensionless form of Problem 1. 
Remark. We non-dimensionalize Problem 1 as above because it preserves the form of the problem and makes the roles of the original material constants explicit in our analysis. However, it is more common to derive a dimensionless form of the problem by using the viscosity instead of the density to eliminate the mass dimension of the relevant variables. To do so, define $\boldsymbol{x}^{*}, t^{*}, \boldsymbol{v}^{*}$ and $\boldsymbol{w}^{*}$ as before but let $\boldsymbol{E}^{*}=L\left(\mu_{0} V\right)^{-1} \hat{\boldsymbol{E}}, p^{*}=L\left(\mu_{0} V\right)^{-1} \hat{p}, \boldsymbol{f}^{*}=\rho L^{2}\left(\mu_{0} V\right)^{-1} \boldsymbol{f}, K^{*}=L \mu_{0}^{-1} \hat{K}$, $\omega=1-\lambda_{2} \lambda_{1}^{-1}$ (retardation parameter), Re $=\rho L V \mu_{0}^{-1}$ (Reynolds number) and We $=\lambda_{1} V L^{-1}$ (Weissenberg number). Then, omitting the asterisks, one obtains the following dimensionless system:

$$
\begin{aligned}
\boldsymbol{E}+\mathrm{We}\left(\boldsymbol{v} \cdot \nabla \boldsymbol{E}+P_{a}(\nabla \boldsymbol{v}, \boldsymbol{E})\right)-\omega \boldsymbol{A} & =0 & & \text { in } \Omega, \\
\operatorname{Re} \boldsymbol{v} \cdot \nabla \boldsymbol{v}-(1-\omega) \triangle \boldsymbol{v}+\nabla p-\operatorname{div} \boldsymbol{E} & =\boldsymbol{f} & & \text { in } \Omega, \\
\operatorname{div} \boldsymbol{v} & =0 & & \text { in } \Omega, \\
{[(\boldsymbol{E}+(1-\omega) \boldsymbol{A}) \boldsymbol{n}]_{\tau}+K \boldsymbol{v} } & =K \boldsymbol{w} & & \text { on } \Gamma, \\
\boldsymbol{v} \cdot \boldsymbol{n} & =0 & & \text { on } \Gamma .
\end{aligned}
$$

Here, $\omega=1$ in the Maxwell case and $0<\omega<1$ in the Jeffreys case.

\subsection{Main result}

We shall prove that for sufficiently small data, Problem 1 has a strong solution:

Theorem 1. Let $\Omega$ be a bounded domain in $\mathbb{R}^{3}$, locally situated on one side of its boundary, $\Gamma$, with $\Gamma \in C^{4}$. Furthermore, let $\boldsymbol{f} \in \boldsymbol{H}^{1}(\Omega)$ and $\boldsymbol{w} \in \boldsymbol{H}^{3 / 2}(\Gamma)$ with $\boldsymbol{w} \cdot \boldsymbol{n}=0$ on $\Gamma$. Then there are positive constants $\Lambda=\Lambda\left(\Omega, \nu_{n}, K\right), C_{T}=C_{T}(\Omega)$ and $C_{*}=C_{*}(\Omega)$ such that the following hold: If

$$
0<\nu_{e}<\Lambda \nu_{n}
$$

there is a positive constant $D_{*}=D_{*}\left(\Omega, \lambda_{1}, \nu_{n}, \nu_{e}, K\right)<\left(\lambda_{1} C_{T} C_{*}\right)^{-2}$ with the property that if

$$
D:=\|\boldsymbol{f}\|_{1,2}+K\|\boldsymbol{w}\|_{3 / 2,2, \Gamma} \leq D_{*}
$$

then Problem 1 has a solution $(\boldsymbol{E}, \boldsymbol{v}, p) \in H_{T}^{2}(\Omega) \times \boldsymbol{H}^{3}(\Omega) \times \bar{H}^{2}(\Omega)$ which satisfies the a priori estimates

$$
\begin{aligned}
\|\boldsymbol{E}\|_{2,2} & \leq \frac{2 \nu_{e} C_{*} \sqrt{D}}{1-\lambda_{1} C_{T} C_{*} \sqrt{D}}, \\
\|\boldsymbol{v}\|_{3,2}+\frac{1}{\nu_{n}}\|p\|_{2,2} & \leq C_{*} \sqrt{D} .
\end{aligned}
$$

Remark. (a) In Section 5 we give sufficient conditions for the local uniqueness of the solution for small data.

(b) The unusual a priori estimates (1.23)-(1.24) result from steps in the proof of Theorem 1 aimed at maximizing $D_{*}$. One can derive a priori estimates which are linear in $D$ by imposing a stronger restriction on $D$; see the remark after the proof of Theorem 1.

(c) We assume that $\mu_{0}$ and $\lambda_{2}$ are positive because our method of proof requires that $\mu_{n}>0$. Then the "Newtonian part", $2 \mu_{n} \boldsymbol{D}$, of the extra-stress is nonzero and we can apply results from the theory of Newtonian fluids to the subproblem (1.12)-(1.15) in a fixed point formulation of Problem 1. Moreover, in order to obtain suitable estimates in this approach, $\mu_{e} / \mu_{n}=\lambda_{1} / \lambda_{2}-1(=\omega /(1-\omega)$ in (1.16)(1.20)) must be sufficiently small; this is assumption $(1.21)_{2}$. Thus, the fluid is 
weakly viscoelastic, as in [7, 9]. This restriction on the fluid model is a consequence of using equation (1.6) - which leads to a simple fixed point formulation of the problem - and is not necessary for solving the original problem. Renardy [6] and Talhouk [13] use a different approach to prove the solvability of the problem with a no-slip boundary condition $(\boldsymbol{v}=0$ on $\Gamma)$ for both the Maxwell model and the Jeffreys model without assumption $(1.21)_{2}$. The basic idea is to apply the divergence operator to equation (1.1), substitute the resulting expression for $\operatorname{div} \boldsymbol{S}$ into the equation of motion and then solve the resulting system by a fixed point method.

\section{Notation}

Let $\Omega$ be a bounded domain in $\mathbb{R}^{3}$ that lies on one side of its boundary, $\Gamma$. The smoothness of $\Gamma$ will be specified by writing, for example, $\Gamma \in C^{2,1}$ to indicate that $\Gamma$ is of class $C^{2,1}$ (Lipschitz continuous second derivatives). We call $\Omega$ rotationally symmetric if it has an axis of symmetry.

For $q \in[1, \infty], L^{q}(\Omega)$ denotes the standard Lebesgue space and $\bar{L}^{q}(\Omega):=\{f \in$ $\left.L^{q}(\Omega): \int_{\Omega} f(x) \mathrm{d} x=0\right\}$. Furthermore, $\boldsymbol{L}^{q}(\Omega)$ denotes a space of vector fields and $L_{T}^{q}(\Omega):=\left\{T=T_{i j} \boldsymbol{e}_{i} \otimes \boldsymbol{e}_{j}: T_{i j} \in L^{q}(\Omega), 1 \leq i, j \leq 3\right\} \equiv L^{q}(\Omega)^{9}$ denotes a space of tensor fields ( $\boldsymbol{e}_{i}$ denotes the unit vector along the $x_{i}$-axis, $i=1,2,3$, and we use the convention of summation over repeated indices). The spaces $L^{q}(\Gamma), \boldsymbol{L}^{q}(\Gamma)$ and $L_{T}^{q}(\Gamma)$ are defined analogously. The norms in $L^{q}(\Omega), \boldsymbol{L}^{q}(\Omega)$ and $L_{T}^{q}(\Omega)$ are denoted by $\|\cdot\|_{q}$, and the norms in $L^{q}(\Gamma), \boldsymbol{L}^{q}(\Gamma)$ and $L_{T}^{q}(\Gamma)$ are denoted by $\|\cdot\|_{q, \Gamma}$. The inner products in $L^{2}(\Omega), \boldsymbol{L}^{2}(\Omega)$ and $L_{T}^{2}(\Omega)$ are denoted by $(\cdot, \cdot)$, and the inner products in $L^{2}(\Gamma), \boldsymbol{L}^{2}(\Gamma)$ and $L_{T}^{2}(\Gamma)$ are denoted by $(\cdot, \cdot)_{\Gamma}$.

For $m \in \mathbb{N}$ and $q \in[1, \infty), W^{m, q}(\Omega)$ denotes the standard Sobolev space with the norm $\|\cdot\|_{m, q}$ (see, e.g., [73, p. 59]). For convenience we define $\|\cdot\|_{0, q}:=\|\cdot\|_{q}$. Furthermore, we denote the Hilbert space $W^{m, 2}(\Omega)$ and its inner product by $H^{m}(\Omega)$ and $(\cdot, \cdot)_{m}$, respectively, and let $\bar{H}^{m}(\Omega):=H^{m}(\Omega) \cap \bar{L}^{1}(\Omega)$. If $\Gamma \in C^{m-1,1}$, we denote the space of traces on $\Gamma$ associated with $H^{m}(\Omega)$ by $H^{m-1 / 2}(\Gamma)$, its inner product by $(\cdot, \cdot)_{m-1 / 2, \Gamma}$ and its norm by $\|\cdot\|_{m-1 / 2,2, \Gamma}$. As above we use bold letters and the subscript $T$ to denote the corresponding spaces of vector-valued functions and tensor-valued functions, respectively, and denote their norms as in the scalar case. Furthermore, for $m \in \mathbb{N}$,

$$
\boldsymbol{H}_{\tau}^{m}(\Omega):=\left\{\boldsymbol{v} \in \boldsymbol{H}^{m}(\Omega): \boldsymbol{v} \cdot \boldsymbol{n}=0 \text { on } \Gamma\right\},
$$

where $\boldsymbol{n}$ is the outer unit normal vector on $\Gamma$ and the boundary condition is meant in the sense of traces, and

$$
\boldsymbol{V}^{m}(\Omega):=\left\{\boldsymbol{v} \in \boldsymbol{H}_{\tau}^{m}(\Omega): \operatorname{div} \boldsymbol{v}=0 \text { a.e. in } \Omega\right\} .
$$

For $m=0,1,2, \ldots, C^{m}(\Omega)$ denotes the linear space of all continuous functions on $\Omega$ with continuous derivatives of all orders up to $m$ on $\Omega$, and $C^{m}(\bar{\Omega})$ denotes the space of all functions in $C^{m}(\Omega)$ that, together with their derivatives of order up to $m$, are bounded and uniformly continuous on $\Omega$. The standard norm in $C^{m}(\bar{\Omega})$ is denoted by $\|\cdot\|_{C^{m}}$ (see, e.g., $[73$, p. 10]).

If $X$ and $Y$ are normed linear spaces, $R>0, D \subseteq X$ and $L: D \rightarrow Y$ is a bounded linear operator, then $\bar{B}(X, R):=\left\{x \in X:\|x\|_{X} \leq R\right\}$ and $\|L\|^{*}:=$ $\sup \left\{\|L x\|_{Y}: x \in D,\|x\|_{X}=1\right\}$. 
In inequalities, $C(\Omega, \ldots)$ denotes a generic positive constant which depends at most on the quantities given in brackets and may have different values even in the same calculation. Fixed constants are denoted by other symbols or by the addition of a subscript or superscript. In particular, by virtue of the Sobolev imbeddings $H^{1}(\Omega) \hookrightarrow L^{q}(\Omega), q \in[2,6]$, and $H^{2}(\Omega) \hookrightarrow C^{0}(\bar{\Omega})$ (for which it is sufficient that $\Gamma \in C^{2}$; see [73, pp. 84-5]), there exist constants $K_{q}=K_{q}(\Omega, q), q \in[2,6]$, and $K_{0}=K_{0}(\Omega)$ such that

$$
\begin{aligned}
\|u\|_{q} \leq K_{q}\|u\|_{1,2} & \text { for all } u \in H^{1}(\Omega), \\
\|u\|_{C^{0}} \leq K_{0}\|u\|_{2,2} & \text { for all } u \in H^{2}(\Omega) .
\end{aligned}
$$

By repeated application of Hölder's inequality and inequalities (2.1)-(2.2), one can show that there exist constants $K_{7}=K_{7}(\Omega)$ and $K_{8}=K_{8}(\Omega)$ such that

$$
\begin{array}{ll}
\|u v\|_{1,2} \leq K_{7}\|u\|_{2,2}\|v\|_{1,2} & \text { for all } u \in H^{2}(\Omega), v \in H^{1}(\Omega), \\
\|u v\|_{2,2} \leq K_{8}\|u\|_{2,2}\|v\|_{2,2} & \text { for all } u, v \in H^{2}(\Omega) .
\end{array}
$$

These inequalities can be derived in more than one way (see, e.g., [73, p. 106] for a proof of (2.4)) but one approach yields $K_{7}=\sqrt{K_{0}^{2}+K_{4}^{4}}$ and $K_{8}=3 K_{7}$; we shall assume that $K_{7} \leq K_{8}$.

The following list gives special constants and the numbers of the equations where they are introduced.

$\begin{array}{cl}\Lambda & (1.21),(4.11) \\ C_{*} & (1.24),(4.12) \\ K_{0} & (2.2) \\ K_{8} & (2.4) \\ C_{1}, \gamma_{1} & (3.4),(\mathrm{A} .13) \\ \gamma_{3} & (\mathrm{~A} .7) \\ C_{5}, C_{5}^{*} & (3.11) \\ c_{2} & (3.15) \\ c_{1}^{*} & (3.17) \\ C_{T} & (4.2) \\ \delta & (4.2),(4.13) \\ C_{8} & (4.19)\end{array}$

$\begin{array}{cl}D, D_{*} & (1.22),(4.12) \\ K_{q}, q \in[2,6] & (2.1) \\ K_{7} & (2.3) \\ C_{2}, \gamma_{2} & (3.1),(\mathrm{A} .10) \\ \alpha, \theta & (\mathrm{A} .3),(\mathrm{A} .12) \\ C_{4}, C_{4}^{*}, \nu_{K} & (3.10) \\ c_{0} & (3.14) \\ N_{1} & (3.16) \\ C_{7} & (3.18) \\ C_{6} & (4.8) \\ Q & (4.10) \\ C_{9} & (4.20)\end{array}$

\section{Auxiliary results}

The proof of Theorem 1 is based on a fixed point argument in which Problem 1 is decomposed into a tensor transport equation and a Stokes system with Navier slip boundary conditions. Lemmas 1 and 2 establish the solvability of a corresponding transport equation and Stokes system, respectively, and give a priori estimates for their solutions. The proof of Lemma 1 is given in the Appendix.

Lemma 1. Let $\Omega$ be a bounded domain in $\mathbb{R}^{3}$ that lies on one side of its boundary $\Gamma \in C^{2}$, let $\boldsymbol{v} \in \boldsymbol{V}^{3}(\Omega)$ and $\boldsymbol{F} \in H_{T}^{2}(\Omega)$, and let $M: H_{T}^{2}(\Omega) \rightarrow H_{T}^{2}(\Omega)$ be a bounded linear operator. Then the following hold:

(a) There is a positive constant $C_{2}=C_{2}(\Omega)$ with the property that if

$$
\gamma_{2}:=C_{2}\|\nabla \boldsymbol{v}\|_{2,2}+\|M\|^{*}<1
$$


then there exists a unique tensor $\boldsymbol{E} \in H_{T}^{2}(\Omega)$ such that

$$
\boldsymbol{E}+\boldsymbol{v} \cdot \nabla \boldsymbol{E}+M \boldsymbol{E}=\boldsymbol{F} \quad \text { in } \Omega \text {. }
$$

Moreover,

$$
\|\boldsymbol{E}\|_{2,2} \leq \frac{\|\boldsymbol{F}\|_{2,2}}{1-\gamma_{2}}
$$

(b) Let $\boldsymbol{E}$ be as in part (a) and in addition suppose that $M$ is bounded in the topology of $H_{T}^{1}(\Omega)$, i.e., $\|M\|_{1}^{*}:=\sup \left\{\|M \boldsymbol{U}\|_{1,2}: \boldsymbol{U} \in H_{T}^{2}(\Omega),\|\boldsymbol{U}\|_{1,2}=1\right\}$ is finite. Then there is a constant $C_{1}=C_{1}(\Omega) \in\left(0, C_{2}\right)$ with the property that if

$$
\gamma_{1}:=C_{1}\|\nabla \boldsymbol{v}\|_{2,2}+\|M\|_{1}^{*}<1
$$

then

$$
\|\boldsymbol{E}\|_{1,2} \leq \frac{\|\boldsymbol{F}\|_{1,2}}{1-\gamma_{1}}
$$

(c) Let $\boldsymbol{E}$ be as in part (a) and in addition suppose that $\boldsymbol{F}$ is symmetric and $(M \boldsymbol{U})^{T} \in\left\{M \boldsymbol{U}, M\left(\boldsymbol{U}^{T}\right)\right\}$ for all $\boldsymbol{U} \in H_{T}^{2}(\Omega)$. Then $\boldsymbol{E}$ is symmetric.

Lemma 2. (a) Suppose that $\Omega$ is a bounded domain in $\mathbb{R}^{3}$ with boundary $\Gamma \in C^{2,1}$ (or $\Gamma \in C^{3}$ ), $\nu>0, K>0, \boldsymbol{a} \in \boldsymbol{L}^{2}(\Omega)$ and $\boldsymbol{b} \in \boldsymbol{H}^{1 / 2}(\Gamma)$ with $\boldsymbol{b} \cdot \boldsymbol{n}=0$ on $\Gamma$. Then the Stokes system

$$
\begin{aligned}
-\nu \Delta \boldsymbol{v}+\nabla p & =\boldsymbol{a} & & \text { in } \Omega, \\
\operatorname{div} \boldsymbol{v} & =0 & & \text { in } \Omega, \\
\nu[\boldsymbol{A}(\boldsymbol{v}) \boldsymbol{n}]_{\tau}+K \boldsymbol{v} & =\boldsymbol{b} & & \text { on } \Gamma, \\
\boldsymbol{v} \cdot \boldsymbol{n} & =0 & & \text { on } \Gamma,
\end{aligned}
$$

has a unique solution $(\boldsymbol{v}, p) \in \boldsymbol{H}^{2}(\Omega) \times \bar{H}^{1}(\Omega)$ and there is a constant $C_{4}=$ $C_{4}(\Omega, \nu, K)>0$ such that

$$
\nu\|\boldsymbol{v}\|_{2,2}+\|p\|_{1,2} \leq C_{4}\left(\|\boldsymbol{a}\|_{2}+\|\boldsymbol{b}\|_{1 / 2,2, \Gamma}\right) .
$$

Moreover, if $\Omega$ is not rotationally symmetric, there are positive constants $C_{4}^{*}=$ $C_{4}^{*}(\Omega)$ and $\nu_{K}=\nu_{K}(K)$ such that $C_{4} \leq C_{4}^{*}$ if $\nu \geq \nu_{K}$.

(b) Suppose, in addition, that $m \in \mathbb{N}, \Gamma \in C^{m+3}, \boldsymbol{a} \in \boldsymbol{H}^{m}(\Omega)$ and $\boldsymbol{b} \in \boldsymbol{H}^{m+1 / 2}(\Gamma)$. Then $(\boldsymbol{v}, p) \in \boldsymbol{H}^{m+2}(\Omega) \times \bar{H}^{m+1}(\Omega)$ and there is a constant $C_{5}=C_{5}(\Omega, m, K / \nu)$ such that

$$
\nu\|\boldsymbol{v}\|_{m+2,2}+\|p\|_{m+1,2} \leq C_{5}\left(\|\boldsymbol{a}\|_{m, 2}+\|\boldsymbol{b}\|_{m+1 / 2,2, \Gamma}\right) .
$$

Moreover, if $\Omega$ is not rotationally symmetric, $C_{5} \leq C_{5}^{*}=C_{5}^{*}(\Omega, m)$ if $\nu \geq \nu_{K}$.

Proof. (a) Problem (3.6)-(3.9) is a particular case of the generalized Stokes system studied by Beirão da Veiga [74] (we have $\mu=\lambda=0, g \equiv 0, a \equiv 0$ in the notation of [74]) and the existence and uniqueness of a solution $(\boldsymbol{v}, p) \in \boldsymbol{H}^{2}(\Omega) \times \bar{H}^{1}(\Omega)$ are immediate consequences of Theorems 1.1-1.2 of [74]. (Theorem 1.1, which deals with weak solutions, requires that $\Gamma \in C^{1,1}$. Theorem 1.2 of [74] is stated with the hypothesis $\Gamma \in C^{2,1}$ and for simplicity some calculations in its proof are performed with $\Gamma \in C^{3}$, but it is mentioned (see [74, pp. 1096, 1102]) that $\Gamma \in W^{3,3}$ is sufficient.) Moreover, Theorem 1.2 of [74] gives the a priori estimate

$$
\|\boldsymbol{v}\|_{2,2}+\|p\|_{1,2} \leq C(\Omega, \nu, K)\left(\|\boldsymbol{a}\|_{2}+\|\boldsymbol{b}\|_{1 / 2,2, \Gamma}\right)
$$

but does not specify the dependence of the constant on $\nu$ and $K$. We shall show how estimate (3.10) can be derived from inequality (3.12) and calculations in [74]. To 
avoid a lengthy repetition of these calculations, we shall refer directly to equations in [74].

For problem (3.6)-(3.9), the definition of a weak solution in [74] means that $(\boldsymbol{v}, p) \in \boldsymbol{H}_{\tau}^{1}(\Omega) \times L^{1}(\Omega)$ and

$$
\nu B_{1}(\boldsymbol{v}, \boldsymbol{\phi})-(p, \operatorname{div} \boldsymbol{\phi})+K(\boldsymbol{v}, \boldsymbol{\phi})_{\Gamma}+(\operatorname{div} \boldsymbol{v}, \psi)=(\boldsymbol{a}, \boldsymbol{\phi})+(\boldsymbol{b}, \boldsymbol{\phi})_{\Gamma}
$$

for all $(\phi, \psi) \in \boldsymbol{H}_{\tau}^{1}(\Omega) \times L^{1}(\Omega)$, where $B_{1}$ is the bilinear form defined by

$$
B_{1}(\boldsymbol{v}, \phi)=2(\boldsymbol{D}(\boldsymbol{v}), \boldsymbol{D}(\boldsymbol{\phi}))-(\operatorname{div} \boldsymbol{v}, \operatorname{div} \phi), \quad \boldsymbol{v}, \boldsymbol{\phi} \in \boldsymbol{H}^{1}(\Omega) .
$$

This implies that $\operatorname{div} \boldsymbol{v} \equiv 0$ and

$$
\nu B_{1}(\boldsymbol{v}, \boldsymbol{v})+K\|\boldsymbol{v}\|_{2, \Gamma}^{2}=(\boldsymbol{a}, \boldsymbol{v})+(\boldsymbol{b}, \boldsymbol{v})_{\Gamma} .
$$

By Lemma 2.2 of [74] with $\mu=0$ and $\nu=1$, there is a positive constant $c_{0}=c_{0}(\Gamma)$ such that

$$
\|\nabla \phi\|_{2}^{2} \leq B_{1}(\phi, \phi)+c_{0}\|\phi\|_{2, \Gamma}^{2} \quad \text { for all } \boldsymbol{\phi} \in \boldsymbol{H}_{\tau}^{1}(\Omega) .
$$

Furthermore, recall (see, e.g., $[75$, pp. 51,56$])$ that $\|\nabla(\cdot)\|_{2}$ is a norm on $\boldsymbol{H}_{\tau}^{1}(\Omega)$ and is equivalent to $\|\cdot\|_{1,2}$; there is a constant $c_{2}=c_{2}(\Omega)$ such that

$$
\|\phi\|_{1,2} \leq c_{2}\|\nabla \phi\|_{2} \quad \text { for all } \boldsymbol{\phi} \in \boldsymbol{H}_{\tau}^{1}(\Omega) .
$$

There are two cases to consider.

Case 1: $\Omega$ is not rotationally symmetric. In this case it follows from the proof of Lemma 2.3 of [74] with $\mu=0$ and $\nu=1$ that for every $\varepsilon>0$ there exists $N_{1}=N_{1}(\Omega, \varepsilon)>0$ such that

$$
\|\phi\|_{2, \Gamma}^{2} \leq \varepsilon\|\nabla \phi\|_{2}^{2}+N_{1} B_{1}(\phi, \phi) \text { for all } \boldsymbol{\phi} \in \boldsymbol{H}_{\tau}^{1}(\Omega) .
$$

(Note that the number $N$ in [74, eq. (2.22)] depends on $\nu: B=\nu B_{1}$ and $N=N_{1} / \nu$.) Thus, by inequalities (3.14) and (3.16) with $\varepsilon=\varepsilon_{0}:=\left(2 c_{0}\right)^{-1}$,

$$
c_{1}^{*}\|\nabla \phi\|_{2}^{2} \leq B_{1}(\phi, \phi) \quad \text { for all } \boldsymbol{\phi} \in \boldsymbol{H}_{\tau}^{1}(\Omega)
$$

where $c_{1}^{*}=c_{1}^{*}(\Omega)=\left(2\left[1+c_{0} N_{1}\left(\varepsilon_{0}\right)\right]\right)^{-1}$ (the constant in [74, Lemma 2.3] is $\left.c_{1}=\nu c_{1}^{*}\right)$. Hence, by applying inequalities (3.15) and (3.17), the Cauchy-Schwarz inequality and the trace theorem to equation (3.13), we get

$$
\nu\|\boldsymbol{v}\|_{1,2} \leq c_{2} \nu\|\nabla \boldsymbol{v}\|_{2} \leq \frac{C_{7}}{c_{1}^{*}}\left(\|\boldsymbol{a}\|_{2}+\|\boldsymbol{b}\|_{2, \Gamma}\right) .
$$

with $C_{7}=C_{7}(\Omega)=\max \left\{1, C_{t}\right\} c_{2}^{2}$, where $C_{t}=C_{t}(\Omega)$ is the constant from the trace theorem.

Note that the derivation of estimate (3.18) does not exploit the fact that $K$ is strictly positive. It can be improved as follows. If $K / \nu \geq c_{0}$, it is not necessary to use inequality (3.16); see Case 2 below. If $K / \nu<c_{0}$, it follows from inequalities (3.14)(3.16) with $\varepsilon=\varepsilon_{K}:=\left(2\left[c_{0}-K / \nu\right]\right)^{-1}$ that

$$
\hat{c}_{1}\|\nabla \phi\|_{2}^{2} \leq B_{1}(\phi, \phi)+\frac{K}{\nu}\|\phi\|_{2, \Gamma}^{2} \quad \text { for all } \boldsymbol{\phi} \in \boldsymbol{H}_{\tau}^{1}(\Omega),
$$

where $\hat{c}_{1}:=\left(2\left[1+\left(c_{0}-K / \nu\right) N_{1}\left(\varepsilon_{K}\right)\right]\right)^{-1}>c_{1}^{*}$ since $\varepsilon_{K}>\left(2 c_{0}\right)^{-1}$ and since we can assume that $N_{1}$ is a decreasing function of $\varepsilon$. Thus, by proceeding as before, we obtain estimate (3.18) with $\hat{c}_{1}$ in place of $c_{1}^{*}$.

Case 2: $\Omega$ is rotationally symmetric. Inequality (3.14) implies that

$$
\|\nabla \phi\|_{2}^{2} \leq \max \left\{\frac{1}{\nu}, \frac{c_{0}}{K}\right\}\left(\nu B_{1}(\boldsymbol{\phi}, \boldsymbol{\phi})+K\|\boldsymbol{\phi}\|_{2, \Gamma}^{2}\right) \quad \text { for all } \boldsymbol{\phi} \in \boldsymbol{H}_{\tau}^{1}(\Omega) .
$$


Thus, by applying inequalities (3.15) and (3.19) to equation (3.13), we get

$$
\min \left\{\nu, K / c_{0}\right\}\|\boldsymbol{v}\|_{1,2} \leq c_{2} \min \left\{\nu, K / c_{0}\right\}\|\nabla \boldsymbol{v}\|_{2} \leq C_{7}\left(\|\boldsymbol{a}\|_{2}+\|\boldsymbol{b}\|_{2, \Gamma}\right) .
$$

Now, in both cases it follows from equations (3.6)-(3.9) that $(\nu \boldsymbol{v}, p)$ is the solution of the Stokes system

$$
\begin{aligned}
-\Delta \boldsymbol{u}+\nabla p & =\boldsymbol{a} & & \text { in } \Omega, \\
\operatorname{div} \boldsymbol{u} & =0 & & \text { in } \Omega, \\
{[\boldsymbol{A}(\boldsymbol{u}) \boldsymbol{n}]_{\tau}+\boldsymbol{u} } & =\boldsymbol{b}+(\nu-K) \boldsymbol{v} & & \text { on } \Gamma, \\
\boldsymbol{u} \cdot \boldsymbol{n} & =0 & & \text { on } \Gamma,
\end{aligned}
$$

which is of the form (3.6)-(3.9) with $\nu=1$ and $K=1$ in the left-hand side terms. Thus, in Case 1 it follows from estimates (3.12) and (3.18) that

$$
\begin{aligned}
\nu\|\boldsymbol{v}\|_{2,2}+\|p\|_{1,2} & \leq C(\Omega)\left(\|\boldsymbol{a}\|_{2}+\|\boldsymbol{b}\|_{1 / 2,2, \Gamma}+|\nu-K| \cdot\|\boldsymbol{v}\|_{1 / 2,2, \Gamma}\right) \\
& \leq C(\Omega)(1+|1-K / \nu|)\left(\|\boldsymbol{a}\|_{2}+\|\boldsymbol{b}\|_{1 / 2,2, \Gamma}\right),
\end{aligned}
$$

which gives estimate (3.10). Choose any $\xi>0$ (e.g., $\xi=0.5$ ) and let $\nu_{K}=\xi K$. Then $|1-K / \nu| \leq \xi_{1}:=\max \{1,1 / \xi-1\}$ if $\nu \geq \nu_{K}$. Thus, we can set $C_{4}^{*}=$ $C(\Omega) \max \{2,1 / \xi\}$.

In Case 2, a similar application of estimates (3.12) and (3.20) yields estimate (3.25) if $\nu \leq K / c_{0}$. However, if $\nu>K / c_{0}$, we obtain

$$
\nu\|\boldsymbol{v}\|_{2,2}+\|p\|_{1,2} \leq C(\Omega)\left(1+c_{0}|\nu / K-1|\right)\left(\|\boldsymbol{a}\|_{2}+\|\boldsymbol{b}\|_{1 / 2,2, \Gamma}\right) .
$$

(b) It follows from part (a) and the classical theorem of Agmon, Douglis and Nirenberg [76, Theorem 10.5] that $(\boldsymbol{v}, p) \in \boldsymbol{H}^{m+2}(\Omega) \times \bar{H}^{m+1}(\Omega)$ and

$$
\|\boldsymbol{v}\|_{m+2,2}+\|p\|_{m+1,2} \leq C(\Omega, m, \nu, K)\left(\|\boldsymbol{a}\|_{m, 2}+\|\boldsymbol{b}\|_{m+1 / 2,2, \Gamma}\right) .
$$

Furthermore, application of the same theorem to the system (3.21)-(3.24) yields the estimate

$$
\nu\|\boldsymbol{v}\|_{j+2,2}+\|p\|_{j+1,2} \leq C(\Omega, j)\left(\|\boldsymbol{a}\|_{j, 2}+\|\boldsymbol{b}\|_{j+1 / 2,2, \Gamma}+|\nu-K| \cdot\|\boldsymbol{v}\|_{j+1 / 2,2, \Gamma}\right)
$$

for $j=1,2, \ldots, m$. Thus, in Case 1 , it follows from this inequality with $j=1$, the trace theorem and inequality (3.25) that

$$
\begin{aligned}
\nu\|\boldsymbol{v}\|_{3,2}+\|p\|_{2,2} \leq & C(\Omega)\left(\|\boldsymbol{a}\|_{1,2}+\|\boldsymbol{b}\|_{3 / 2,2, \Gamma}+|\nu-K| \cdot\|\boldsymbol{v}\|_{3 / 2,2, \Gamma}\right) \\
\leq & C(\Omega)[1+|1-K / \nu|(1+|1-K / \nu|)] \cdot \\
& \cdot\left(\|\boldsymbol{a}\|_{1,2}+\|\boldsymbol{b}\|_{3 / 2,2, \Gamma}\right) .
\end{aligned}
$$

By repeating this argument with $j=2,3, \ldots, m$ we obtain

$$
\begin{aligned}
& \nu\|\boldsymbol{v}\|_{m+2,2}+\|p\|_{m+1,2} \leq C(\Omega, m)\left(\|\boldsymbol{a}\|_{m, 2}+\|\boldsymbol{b}\|_{m+1 / 2,2, \Gamma}\right) \cdot \\
& \cdot\left(1+|1-K / \nu|+|1-K / \nu|^{2}+\cdots+|1-K / \nu|^{m-1}\right),
\end{aligned}
$$

which gives estimate (3.11). Thus, with $\nu_{K}$ and $\xi_{1}$ as in the proof of part (a) (below $(3.25)$ ), we can take $C_{5}^{*}=C(\Omega, m)\left(1+\xi_{1}+\xi_{1}^{2}+\cdots+\xi_{1}^{m-1}\right)$.

In Case 2, a similar argument leads to estimates (3.27)-(3.28) and (3.11) if $\nu \leq K / c_{0}$. If $\nu>K / c_{0}$, we apply estimate (3.26) instead of (3.25) and obtain

$$
\begin{aligned}
\nu\|\boldsymbol{v}\|_{m+2,2}+\|p\|_{m+1,2} \leq C(\Omega, m)\left(\|\boldsymbol{a}\|_{m, 2}+\|\boldsymbol{b}\|_{m+1 / 2,2, \Gamma}\right) \cdot \\
\cdot\left(1+|1-K / \nu|+\cdots+|1-K / \nu|^{m-2}+(\nu / K)|1-K / \nu|^{m-1}\right),
\end{aligned}
$$


which is also of the form (3.11).

Remark. If $\partial \Omega$ consists of more than one separate component, say $\partial \Omega=\Gamma \cup \Sigma$, where $\Gamma$ and $\Sigma$ are closed manifolds without boundary and $\operatorname{dist}(\Gamma, \Sigma)>0$, one can impose the no-slip boundary condition on $\Sigma$ to avoid the complications that arise when $\Omega$ is rotationally symmetric. In particular, since there is a positive constant $C=C(\Omega, \Sigma)$ such that $C\|\boldsymbol{v}\|_{1,2} \leq\|\boldsymbol{D}(\boldsymbol{v})\|_{2}$ for all $\boldsymbol{v} \in \boldsymbol{H}^{1}(\Omega)$ with $\boldsymbol{v}=0$ on $\Sigma$ (see, e.g., [77]), it is not necessary that $K>0$ for coercivity in the Stokes problem if $\Omega$ is rotationally symmetric. Moreover, the regularity results used in the preceding proof remain valid (see [74, p. 1081]).

Lastly, we recall a classical fixed point theorem of Schauder [78].

Lemma 3. Suppose that $V$ is a non-empty, convex and compact subset of a complete metric space $X$ and $\Phi: V \rightarrow X$ is a continuous mapping such that $\Phi(V) \subseteq V$. Then $\Phi$ has a fixed point.

\section{Proof of Theorem 1}

Proof. If $D=0,(\boldsymbol{E}, \boldsymbol{v}, p) \equiv(0,0,0)$ is a solution of Problem 1 and satisfies estimates (1.23)-(1.24). Thus, assume that $D>0$, let $R>0$ (the value of $R$ will be chosen later) and let $V_{R}:=\bar{B}\left(\boldsymbol{V}^{3}(\Omega), R\right)$ and $X:=\boldsymbol{H}^{2}(\Omega)$.

(1) $V_{R}$ is nonempty and convex. To verify that $V_{R}$ is compact in $\boldsymbol{H}^{2}(\Omega)$, let $\left(\boldsymbol{u}_{n}\right)$ be any sequence in $V_{R}$. Then $\left(\boldsymbol{u}_{n}\right)$ is bounded in the Hilbert space $\boldsymbol{H}^{3}(\Omega)$ and therefore has a subsequence $\left(\boldsymbol{u}_{n_{k}}\right)$ which converges weakly to some $\boldsymbol{u}$ in $\boldsymbol{H}^{3}(\Omega)$. Hence, $\boldsymbol{u}_{n_{k}} \longrightarrow \boldsymbol{u}$ in $\boldsymbol{H}^{2}(\Omega)$ because $\boldsymbol{H}^{3}(\Omega) \hookrightarrow \hookrightarrow \boldsymbol{H}^{2}(\Omega)$, and $\boldsymbol{u} \in V_{R}$ because $\|\boldsymbol{u}\|_{3,2} \leq \liminf \left\|\boldsymbol{u}_{n_{k}}\right\|_{3,2} \leq R$.

(2) Let $\boldsymbol{u} \in V_{R}$ and consider the tensor transport equation

$$
\boldsymbol{E}+\lambda_{1} \boldsymbol{u} \cdot \nabla \boldsymbol{E}+\lambda_{1} P_{a}(\nabla \boldsymbol{u}, \boldsymbol{E})=\nu_{e} \boldsymbol{A}(\boldsymbol{u}) \quad \text { in } \Omega .
$$

One can show by means of inequality (2.4) and the Cauchy-Schwarz inequality that

$$
\|\boldsymbol{G} \boldsymbol{H}\|_{2,2} \leq K_{8}\|\boldsymbol{G}\|_{2,2}\|\boldsymbol{H}\|_{2,2} \text { for all } \boldsymbol{G}, \boldsymbol{H} \in H_{T}^{2}(\Omega) .
$$

Thus, $P_{a}(\nabla \boldsymbol{u}, \boldsymbol{E}) \in H_{T}^{2}(\Omega)$ and

$$
\left\|P_{a}(\nabla \boldsymbol{u}, \boldsymbol{E})\right\|_{2,2} \leq 2 K_{8}\|\nabla \boldsymbol{u}\|_{2,2}\|\boldsymbol{E}\|_{2,2} \quad \text { for all } \boldsymbol{E} \in H_{T}^{2}(\Omega) .
$$

Hence, the mapping $M: \boldsymbol{E} \mapsto \lambda_{1} P_{a}(\nabla \boldsymbol{u}, \boldsymbol{E})$ defines a bounded linear operator $M: H_{T}^{2}(\Omega) \rightarrow H_{T}^{2}(\Omega)$ and $\|M\|^{*} \leq 2 \lambda_{1} K_{8}\|\nabla \boldsymbol{u}\|_{2,2} \leq 2 \lambda_{1} K_{8} R$. Furthermore, $\boldsymbol{F}:=$ $\nu_{e} \boldsymbol{A}(\boldsymbol{u}) \in H_{T}^{2}(\Omega)$. Assume that $\lambda_{1} C_{T} R<1$, where $C_{T}=C_{T}(\Omega):=C_{2}+2 K_{8}$. Moreover, assume that

$$
R \leq \frac{1-\delta}{\lambda_{1} C_{T}}
$$

for some $\delta \in(0,1)$ (the value of $\delta$ will be chosen later; see (4.13)). Then, by Lemma 1(a), equation (4.1) has a unique solution, $\boldsymbol{E}$, in $H_{T}^{2}(\Omega)$ and

$$
\|\boldsymbol{E}\|_{2,2} \leq \frac{2 \nu_{e}\|\nabla \boldsymbol{u}\|_{2,2}}{1-\lambda_{1} C_{T}\|\nabla \boldsymbol{u}\|_{2,2}} \leq \frac{2 \nu_{e} R}{1-\lambda_{1} C_{T} R} \leq \frac{2 \nu_{e} R}{\delta} .
$$

Furthermore, $\boldsymbol{F}$ is symmetric and it follows from the definition of $P_{a}$ that $(M \boldsymbol{U})^{T}=$ $M\left(\boldsymbol{U}^{T}\right)$ for all $\boldsymbol{U} \in H_{T}^{2}(\Omega)$. Thus, by Lemma 1(c), $\boldsymbol{E}$ is symmetric. 
Next, consider the Stokes system

$$
\begin{aligned}
-\nu_{n} \Delta \boldsymbol{v}+\nabla p & =\boldsymbol{f}+\operatorname{div} \boldsymbol{E}-\boldsymbol{u} \cdot \nabla \boldsymbol{u} & & \text { in } \Omega, \\
\operatorname{div} \boldsymbol{v} & =0 & & \text { in } \Omega, \\
\nu_{n}[\boldsymbol{A}(\boldsymbol{v}) \boldsymbol{n}]_{\tau}+K \boldsymbol{v} & =K \boldsymbol{w}-[\boldsymbol{E n}]_{\tau} & & \text { on } \Gamma, \\
\boldsymbol{v} \cdot \boldsymbol{n} & =0 & & \text { on } \Gamma,
\end{aligned}
$$

where $\boldsymbol{E}$ is the solution of equation (4.1). By Lemma 2, this problem has a unique solution $(\boldsymbol{v}, p) \in \boldsymbol{H}^{3}(\Omega) \times \bar{H}^{2}(\Omega)$. Thus we can define a mapping $\Phi: V_{R} \mapsto \boldsymbol{V}^{3}(\Omega)$ by $\Phi(\boldsymbol{u})=\boldsymbol{v}$. By construction, if $\boldsymbol{v}$ is a fixed point of $\Phi$ then the corresponding triple $(\boldsymbol{E}, \boldsymbol{v}, p)$ is a solution of Problem 1.

(3) The next step is to show that $\Phi\left(V_{R}\right) \subseteq V_{R}$ for suitable choices of $D_{*}$ and $R$. To this end, let $\boldsymbol{u}, \boldsymbol{E}, \boldsymbol{v}$ and $p$ be as in part (2) above. By virtue of the trace theorem, there is a constant $C_{6}=C_{6}(\Omega)$ such that

$$
\|\operatorname{div} \boldsymbol{E}\|_{1,2}+\left\|[\boldsymbol{E} \boldsymbol{n}]_{\tau}\right\|_{3 / 2,2, \Gamma} \leq C_{6}\|\boldsymbol{E}\|_{2,2} .
$$

Furthermore, it follows from inequality (2.3) and the Cauchy-Schwarz inequality that

$$
\|\boldsymbol{u} \cdot \nabla \boldsymbol{u}\|_{1,2} \leq K_{7}\|\boldsymbol{u}\|_{2,2}^{2} .
$$

Thus, by estimates (3.11) (with $m=1$ and $\left.C_{5}=C_{5}\left(\Omega, \nu_{n}, K\right)\right)$ and (4.3),

$$
\begin{aligned}
\|\boldsymbol{v}\|_{3,2}+\frac{1}{\nu_{n}}\|p\|_{2,2} & \leq \frac{C_{5}}{\nu_{n}}\left(C_{6}\|\boldsymbol{E}\|_{2,2}+K_{7}\|\boldsymbol{u}\|_{2,2}^{2}+\|\boldsymbol{f}\|_{1,2}+K\|\boldsymbol{w}\|_{3 / 2,2, \Gamma}\right) \\
& \leq \frac{C_{5}}{\nu_{n}}\left(\frac{2 C_{6} \nu_{e} R}{\delta}+K_{7} R^{2}+D\right) .
\end{aligned}
$$

The right-hand side of (4.9) is less than or equal to $R$ if and only if

$$
D \leq R\left(Q-K_{7} R\right)=\frac{Q^{2}}{4 K_{7}}-K_{7}\left(R-\frac{Q}{2 K_{7}}\right)^{2}, \quad Q:=\frac{\nu_{n}}{C_{5}}-\frac{2 C_{6} \nu_{e}}{\delta} .
$$

Now, $Q>0$ for all $\delta>2 C_{5} C_{6} \nu_{e} / \nu_{n}$. Assume that inequality (1.21) holds with $\Lambda=\left(2 C_{5} C_{6}\right)^{-1}$. Then

$$
0<\frac{2 C_{5} C_{6} \nu_{e}}{\nu_{n}}<1 .
$$

Moreover, for any given $\delta \in I=\left(2 C_{5} C_{6} \nu_{e} / \nu_{n}, 1\right)$, the largest possible value of $D$ in inequality (4.10) is $D_{*}:=Q^{2} /\left(4 K_{7}\right)$ if $R_{*}:=Q /\left(2 K_{7}\right)=\sqrt{D_{*} / K_{7}}=C_{*} \sqrt{D_{*}}$, where $C_{*}=\left(K_{7}\right)^{-1 / 2}$, satisfies inequality (4.2). Thus, in order to maximize $D_{*}$ we choose the largest $\delta \in I$ such that

$$
R_{*}=\frac{1}{2 K_{7}}\left(\frac{\nu_{n}}{C_{5}}-\frac{2 C_{6} \nu_{e}}{\delta}\right) \leq \frac{1-\delta}{\lambda_{1} C_{T}} .
$$

As a function of $\delta$, the left-hand side of this inequality increases monotonely from zero on $I$ and the right-hand side decreases monotonely to zero on $I$. Hence, the largest value of $\delta$ in $I$ for which the inequality holds, is the value at which equality holds, namely

$$
\delta=\frac{1}{2}\left(1-\frac{C_{T} \lambda_{1} \nu_{n}}{2 C_{5} K_{7}}\right)+\frac{1}{2} \sqrt{\left(1-\frac{C_{T} \lambda_{1} \nu_{n}}{2 C_{5} K_{7}}\right)^{2}+\frac{4 C_{6} C_{T} \lambda_{1} \nu_{e}}{K_{7}}} .
$$


With this choice of $\delta$, assume that $0<D \leq D_{*}=Q^{2} /\left(4 K_{7}\right)$ and set $R=\sqrt{D / K_{7}}=$ $C_{*} \sqrt{D}$. (Note that by $(4.10)_{2}$ and (4.13), $D_{*}$ depends only on $\Omega, \lambda_{1}, \nu_{n}, \nu_{e}$ and $K$.) Then $R \leq R_{*}=Q /\left(2 K_{7}\right)$. Hence, it follows from inequality (4.9) that $\boldsymbol{v} \in V_{R}$ :

$$
\begin{aligned}
\|\boldsymbol{v}\|_{3,2}+\frac{1}{\nu_{n}}\|p\|_{2,2} & \leq \frac{C_{5}}{\nu_{n}}\left(\left[\frac{2 C_{6} \nu_{e}}{\delta}-\frac{\nu_{n}}{C_{5}}\right] R+2 K_{7} R^{2}\right)+R \\
& =\frac{C_{5}}{\nu_{n}}\left(2 K_{7} R-Q\right) R+R \leq R .
\end{aligned}
$$

(4) It remains to show that $\Phi$ is continuous in the topology of $\boldsymbol{H}^{2}(\Omega)$. For $i=1,2$, take $\boldsymbol{u}_{i} \in V_{R}$ and let $\boldsymbol{E}_{i}$ denote the solution of equation (4.1) with $\boldsymbol{u}_{i}$ in place of $\boldsymbol{u}$. Let $\boldsymbol{u}:=\boldsymbol{u}_{1}-\boldsymbol{u}_{2}$ and $\boldsymbol{E}:=\boldsymbol{E}_{1}-\boldsymbol{E}_{2}$. Then

$$
\begin{aligned}
\boldsymbol{E}+\lambda_{1} \boldsymbol{u}_{1} \cdot \nabla \boldsymbol{E}+\lambda_{1} P_{a} & \left(\nabla \boldsymbol{u}_{1}, \boldsymbol{E}\right) \\
& =\nu_{e} \boldsymbol{A}(\boldsymbol{u})-\lambda_{1} \boldsymbol{u} \cdot \nabla \boldsymbol{E}_{2}-\lambda_{1} P_{a}\left(\nabla \boldsymbol{u}, \boldsymbol{E}_{2}\right) \text { in } \Omega .
\end{aligned}
$$

Define $M_{1}: H_{T}^{2}(\Omega) \rightarrow H_{T}^{2}(\Omega)$ by $M_{1}(\boldsymbol{E})=\lambda_{1} P_{a}\left(\nabla \boldsymbol{u}_{1}, \boldsymbol{E}\right)$. Then it follows as in part (2) that $\gamma_{2}\left(\boldsymbol{u}_{1}\right)=C_{2}\left\|\nabla \boldsymbol{u}_{1}\right\|_{2,2}+\left\|M_{1}\right\|^{*} \leq \lambda_{1} C_{T} R<1$. Thus, by Lemma 1(a), $\boldsymbol{E}$ is the only solution of equation (4.15) in $H_{T}^{2}(\Omega)$. Furthermore, by applying inequality (2.3) and the Cauchy-Schwarz inequality, one can show that

$$
\left\|\boldsymbol{u} \cdot \nabla \boldsymbol{E}_{2}\right\|_{1,2} \leq K_{7}\left\|\nabla \boldsymbol{E}_{2}\right\|_{1,2}\|\boldsymbol{u}\|_{2,2}
$$

and that $\|\boldsymbol{G} \boldsymbol{H}\|_{1,2} \leq K_{7}\|\boldsymbol{G}\|_{2,2}\|\boldsymbol{H}\|_{1,2}$ for all $\boldsymbol{G} \in H_{T}^{2}(\Omega)$ and all $\boldsymbol{H} \in H_{T}^{1}(\Omega)$. It follows that

$$
\left\|P_{a}\left(\nabla \boldsymbol{u}, \boldsymbol{E}_{2}\right)\right\|_{1,2} \leq 2 K_{7}\left\|\boldsymbol{E}_{2}\right\|_{2,2}\|\nabla \boldsymbol{u}\|_{1,2}
$$

and that $\left\|P_{a}\left(\nabla \boldsymbol{u}_{1}, \boldsymbol{E}\right)\right\|_{1,2} \leq 2 K_{7}\left\|\nabla \boldsymbol{u}_{1}\right\|_{2,2}\|\boldsymbol{E}\|_{1,2}$ for all $\boldsymbol{E} \in H_{T}^{2}(\Omega)$, which implies that

$$
\left\|M_{1}\right\|_{1}^{*} \leq 2 \lambda_{1} K_{7}\left\|\nabla \boldsymbol{u}_{1}\right\|_{2,2} .
$$

Thus, since $C_{1} \leq C_{2}$ and $K_{7} \leq K_{8}$,

$$
\gamma_{1}\left(\boldsymbol{u}_{1}\right)=C_{1}\left\|\nabla \boldsymbol{u}_{1}\right\|_{2,2}+\left\|M_{1}\right\|_{1}^{*} \leq \gamma_{2}\left(\boldsymbol{u}_{1}\right) \leq \lambda_{1} C_{T} R<1 .
$$

Hence, by estimates (3.5) and (4.3) and inequalities (4.16)-(4.17),

$$
\|\boldsymbol{E}\|_{1,2} \leq C_{8}\|\boldsymbol{u}\|_{2,2}, \quad C_{8}:=\frac{2 \nu_{e}}{1-\lambda_{1} C_{T} R}\left(1+\frac{3 \lambda_{1} K_{7} R}{1-\lambda_{1} C_{T} R}\right) .
$$

Next, for $i=1,2$, let $\left(\boldsymbol{v}_{i}, p_{i}\right)$ be the corresponding solution of the Stokes system (4.4)-(4.7). Let $\boldsymbol{v}:=\boldsymbol{v}_{1}-\boldsymbol{v}_{2}$ and $p:=p_{1}-p_{2}$. Then

$$
\begin{aligned}
-\nu_{n} \Delta \boldsymbol{v}+\nabla p & =\operatorname{div} \boldsymbol{E}-\boldsymbol{u}_{1} \cdot \nabla \boldsymbol{u}-\boldsymbol{u} \cdot \nabla \boldsymbol{u}_{2} & & \text { in } \Omega, \\
\operatorname{div} \boldsymbol{v} & =0 & & \text { in } \Omega, \\
\nu_{n}[\boldsymbol{A}(\boldsymbol{v}) \boldsymbol{n}]_{\tau}+K \boldsymbol{v} & =-[\boldsymbol{E n}]_{\tau} & & \text { on } \Gamma, \\
\boldsymbol{v} \cdot \boldsymbol{n} & =0 & & \text { on } \Gamma .
\end{aligned}
$$

By Lemma 2(a), $(\boldsymbol{v}, p)$ is the only solution of this problem in $\boldsymbol{H}^{2}(\Omega) \times \bar{H}^{1}(\Omega)$. Thus, by estimate (3.10), the trace theorem, inequality (2.2), the Cauchy-Schwarz inequality and estimate (4.19),

$$
\begin{gathered}
\nu_{n}\|\boldsymbol{v}\|_{2,2}+\|p\|_{1,2} \leq C_{4}\left(\Omega, \nu_{n}, K\right)\left(C_{9}\|\boldsymbol{E}\|_{1,2}+K_{0}\left\|\boldsymbol{u}_{1}\right\|_{2,2}\|\nabla \boldsymbol{u}\|_{2}\right. \\
\left.+K_{0}\left\|\nabla \boldsymbol{u}_{2}\right\|_{2,2}\|\boldsymbol{u}\|_{2}\right) \\
\leq C_{4}\left(C_{8} C_{9}+2 K_{0} R\right)\|\boldsymbol{u}\|_{2,2}
\end{gathered}
$$


where $C_{9}=C_{9}(\Omega)$. Hence, $\Phi: X \supset V_{R} \rightarrow X$ is (Lipschitz) continuous.

(5) In view of Lemma 3 and paragraphs (1)-(4) above, we conclude that $\Phi$ has a fixed point $\boldsymbol{v} \in V_{R}$. The a priori estimates (1.23)-(1.24) follow from estimates (4.3) and (4.14) and the definition of $R$.

Remark. (a) The simple fixed point formulation of Problem 1 in the proof of Theorem 1 is possible because of the explicit equation, (1.6), for the elastic part of the extra-stress. The advantage of this is evident when one compares it with the fixed point formulations of similar flow problems with wall slip for fluids of grade two and three in [79]-[81], which involve additional quantities and auxiliary problems.

(b) For small values of $D$ it is desirable to have a priori estimates which are linear in $D$ instead of estimates (1.23)-(1.24). To this end, we carry out the proof of Theorem 1 with $R=D / \phi$ for some $\phi>0$. Then, by inequality (4.2), a sufficient condition for the right-hand side of estimate (4.9) to be less than or equal to $R$ is that $X / \delta+Y(1-\delta)+C_{5} \phi / \nu_{n} \leq 1$, where $X:=2 C_{5} C_{6} \nu_{e} / \nu_{n}<1$ by (4.11), $Y:=C_{5} K_{7} /\left(\nu_{n} \lambda_{1} C_{T}\right)$. This holds if, e.g., $\delta=\max \{3 X /(2 X+1), 1-(1-X) /(3 Y)\}$ and $\phi=\nu_{n}(1-X) /\left(3 C_{5}\right)$. Thus, with $0<D \leq D_{*}:=\phi R_{*}, R_{*}:=(1-\delta) /\left(\lambda_{1} C_{T}\right)$, we obtain a solution which satisfies

$$
\|\boldsymbol{E}\|_{2,2} \leq \frac{2 \nu_{e} D}{\delta \phi}, \quad\|\boldsymbol{v}\|_{3,2}+\frac{1}{\nu_{n}}\|p\|_{2,2} \leq \frac{D}{\phi} .
$$

(c) The slip boundary conditions considered in [68]-[71] are of the form

$$
\left[\left(\hat{\boldsymbol{E}}+\mu_{n} \boldsymbol{A}\right) \boldsymbol{n}\right]_{\tau}+\hat{Q}(|\boldsymbol{v}-\boldsymbol{w}|)(\boldsymbol{v}-\boldsymbol{w})=0 \quad \text { on } \Gamma,
$$

where $\hat{Q}:[0, \infty) \rightarrow[0, \infty)$ is given. (In [68]-[71], $\boldsymbol{w} \equiv 0$ and the function $x \mapsto \hat{Q}(x) x$, $x>0$, has a local maximum followed by a local minimum.) To see how the proof of Theorem 1 can be adapted to the corresponding boundary-value problem, let $\hat{Q}(x)=\hat{K}+\hat{S}(x)$ with $\hat{K}>0$ and, for example, $\hat{S}(0)=0$. Then we write boundary condition (4.21) as

$$
\mu_{n}[\boldsymbol{A n}]_{\tau}+\hat{K} \boldsymbol{v}=\hat{K} \boldsymbol{w}-\hat{S}(|\boldsymbol{v}-\boldsymbol{w}|)(\boldsymbol{v}-\boldsymbol{w})-[\hat{\boldsymbol{E}} \boldsymbol{n}]_{\tau} \quad \text { on } \Gamma
$$

and replace boundary condition (4.6) by

$$
\nu_{n}[\boldsymbol{A n}]_{\tau}+K \boldsymbol{v}=K \boldsymbol{w}-S(|\boldsymbol{u}-\boldsymbol{w}|)(\boldsymbol{u}-\boldsymbol{w})-[\boldsymbol{E n}]_{\tau} \quad \text { on } \Gamma,
$$

where $S(x)=\rho^{-1} V^{-1} \hat{S}(V x), x \geq 0$. By imposing suitable restrictions on $S$ (see [81], where the slip condition is also of the form (4.21)), one can then derive an existence and uniqueness result similar to Theorem 1.

\section{Uniqueness of the solution}

Here we show that the solution constructed in the proof of Theorem 1 is locally unique under slightly stronger restrictions on the data.

Theorem 2. Assume that the hypotheses of Theorem 1 and inequalities (1.21)-(1.22) are satisfied. Furthermore, let $D_{0}$ be a positive number such that

$$
0 \leq D \leq D_{0} \leq D_{*}
$$


(a) Suppose that $\Omega$ is not rotationally symmetric. Then there is a constant $\nu_{*}=$ $\nu_{*}\left(\Omega, \lambda_{1}, \nu_{e}, K, D_{0}\right)$ with the property that if $\nu_{n}>\nu_{*}$, then Problem 1 has only one solution $(\boldsymbol{E}, \boldsymbol{v}, p)$ in $H_{T}^{2}(\Omega) \times \boldsymbol{H}^{3}(\Omega) \times \bar{H}^{2}(\Omega)$ that satisfies

$$
\|\boldsymbol{v}\|_{3,2} \leq C_{*} \sqrt{D_{0}} .
$$

(b) Suppose that $\Omega$ is rotationally symmetric. Then there is a constant $K_{*}=$ $K_{*}\left(\Omega, \lambda_{1}, \nu_{e}, D_{0}\right)$ with the property that if $c_{0} \nu_{n}>K \geq K_{*}$, then Problem 1 has only one solution $(\boldsymbol{E}, \boldsymbol{v}, p)$ in $H_{T}^{2}(\Omega) \times \boldsymbol{H}^{3}(\Omega) \times \bar{H}^{2}(\Omega)$ that satisfies inequality (5.1).

(c) In both (a) and (b), the solution satisfies $\|\boldsymbol{v}\|_{3,2} \leq C_{*} \sqrt{D}$ and is therefore isolated if $D<D_{0}$.

Proof. Since $0<D_{0} \leq D_{*}$, we can carry out the proof of Theorem 1 with $D_{0}$ and $R_{0}:=C_{*} \sqrt{D_{0}}$ in place of $D$ (which may be zero) and $R$, respectively. Suppose that $(\boldsymbol{E}, \boldsymbol{v}, p) \in H_{T}^{2}(\Omega) \times \boldsymbol{H}^{3}(\Omega) \times \bar{H}^{2}(\Omega)$ is a solution of Problem 1 and satisfies inequality (5.1). Then, in the notation of the proof of Theorem 1, $v \in V_{R_{0}}$ and it follows from the definition of $\Phi$ and the uniqueness of the solutions of the transport equation (4.1) and the Stokes system (4.4)-(4.7) that $\boldsymbol{v}$ is a fixed point of $\Phi$. Hence, it is sufficient to show that $\Phi$ has only one fixed point in $V_{R_{0}}$.

(a) By inequality (4.20), the property of $C_{4}$ stated in Lemma 2(a), the definition of $C_{8}$ in (4.19) and the definition of $R_{0}, \Phi$ is a contraction on $V_{R_{0}}$ if $\nu_{n}>\nu_{*}:=$ $\max \left\{\nu_{K}, C_{4}^{*}\left(C_{8} C_{9}+2 K_{0} R_{0}\right)\right\}$. (For comparison with part (b) below, note that by estimate (3.25) we can take, for example, $\nu_{K}=K / c_{0}$ and $C_{4}^{*}=C(\Omega) \max \left\{2, c_{0}\right\}$.)

(b) Inequality (4.20) and the definition of $C_{4}$ by inequality (3.26) imply that $\Phi$ is a contraction on $V_{R_{0}}$ if $\nu_{n}>K / c_{0}$ and

$$
\frac{1}{\nu_{n}}+\frac{c_{0}}{K}\left|1-\frac{K}{\nu_{n}}\right|<\frac{1}{C(\Omega)\left(C_{8} C_{9}+2 K_{0} R_{0}\right)} .
$$

Thus, it is sufficient that $c_{0} \nu_{n}>K \geq K_{*}:=c_{0} C(\Omega) \max \left\{2, c_{0}\right\}\left(C_{8} C_{9}+2 K_{0} R_{0}\right)$.

(c) Since $D \leq D_{0}$, the solution in Theorem 1 is the unique solution in either part (a) or (b). Thus, by estimate (1.24), $\boldsymbol{v}$ lies in the interior of $V_{R_{0}}$ if $D<D_{0}$.

Remark. Parts (a) and (b) of Theorem 2 are treated separately because Lemma 2(a) does not ensure that the constant $C_{4}$ in inequality (4.20) is uniformly bounded with respect to $\nu_{n}$ when $\Omega$ is rotationally symmetric.

\section{Appendix}

Transport equations similar to equation (3.2) have been studied by, amongst others, Beirão da Veiga [82, 83], Novotný [84, 85] and Talhouk [13]. Here we give a selfcontained proof of Lemma 1 to show how estimates (3.3) and (3.5) are derived.

Proof. (a) Consider the scalar transport equation

$$
z+\boldsymbol{v} \cdot \nabla z=f \quad \text { in } \Omega,
$$

where $f \in H^{2}(\Omega)$ is given. By Green's formula, $(\boldsymbol{v} \cdot \nabla z, z)=0$ for all $\boldsymbol{v} \in \boldsymbol{V}^{1}(\Omega)$ and all $z \in H^{1}(\Omega)$. It follows that equation (A.1) has at most one solution in $H^{1}(\Omega)$ and therefore the tensor transport equation

$$
\boldsymbol{E}+\boldsymbol{v} \cdot \nabla \boldsymbol{E}=\boldsymbol{F} \text { in } \Omega,
$$


which is a system of independent equations of the type (A.1), has at most one solution in $H_{T}^{1}(\Omega)$. Furthermore, in view of the imbeddings $H^{2}(\Omega) \hookrightarrow C^{0}(\bar{\Omega})$ and $H^{1}(\Omega) \hookrightarrow L^{3}(\Omega)$, it follows from (the proof of) Theorem 5.3 and Remarks 2.1 and 8.1 in [84] (with $k=2, q=2, n=3, a=0, i=1$ ) that there exists a positive constant $\alpha$, which is independent of $\boldsymbol{v}, f$ and the size of $\Omega$, such that if

$$
\alpha \theta<1, \quad \theta:=\|\nabla \boldsymbol{v}\|_{C^{0}}+\left\|\nabla^{2} \boldsymbol{v}\right\|_{0,3},
$$

then equation (A.1) has a unique solution $z \in H^{2}(\Omega)$ and

$$
\|z\|_{2,2} \leq(1-\alpha \theta)^{-1}\|f\|_{2,2} \text {. }
$$

Hence, if inequality (A.3) holds, equation (A.2) has a unique solution $\boldsymbol{E} \in H_{T}^{2}(\Omega)$ and $\|\boldsymbol{E}\|_{2,2} \leq(1-\alpha \theta)^{-1}\|\boldsymbol{F}\|_{2,2}$. In other words, if inequality (A.3) holds, the linear mapping $L: H_{T}^{2}(\Omega) \rightarrow H_{T}^{1}(\Omega)$ defined by $L \boldsymbol{E}=\boldsymbol{E}+\boldsymbol{v} \cdot \nabla \boldsymbol{E}$ is injective, its range contains $H_{T}^{2}(\Omega)$, it has a continuous right inverse $L_{R}^{-1}: H_{T}^{2}(\Omega) \rightarrow H_{T}^{2}(\Omega)$ defined by $L_{R}^{-1}(\boldsymbol{F})=\boldsymbol{E}$, and

$$
\left\|L_{R}^{-1}\right\|^{*} \leq(1-\alpha \theta)^{-1}
$$

Equation (3.2) can now be written as

$$
(L+M) \boldsymbol{E}=L\left(I+L_{R}^{-1} M\right) \boldsymbol{E}=\boldsymbol{F},
$$

where $I$ denotes the identity operator on $H_{T}^{2}(\Omega)$. Now, by inequality (A.5),

$$
\left\|L_{R}^{-1} M\right\|^{*} \leq \gamma_{3}:=(1-\alpha \theta)^{-1}\|M\|^{*} .
$$

Thus, if $\gamma_{3}<1$, i.e., if

$$
\alpha \theta+\|M\|^{*}<1
$$

then it follows from a classical theorem that the mapping $I+L_{R}^{-1} M: H_{T}^{2}(\Omega) \rightarrow$ $H_{T}^{2}(\Omega)$ is a homeomorphism. Moreover, by inequality (A.7),

$$
\left\|\left(I+L_{R}^{-1} M\right)^{-1}\right\|^{*} \leq\left(1-\gamma_{3}\right)^{-1} .
$$

Hence, equation (A.6) has a unique solution, namely $\boldsymbol{E}=\left(I+L_{R}^{-1} M\right)^{-1} L_{R}^{-1} F$, and it follows from estimates (A.5) and (A.9) that

$$
\|\boldsymbol{E}\|_{2,2} \leq\left(1-\alpha \theta-\|M\|^{*}\right)^{-1}\|\boldsymbol{F}\|_{2,2} \text {. }
$$

Now, by inequalities (2.1) and (2.2), $\theta \leq\left(K_{0}+K_{3}\right)\|\nabla \boldsymbol{v}\|_{2,2}$. Thus, with $C_{2}:=$ $\alpha\left(K_{0}+K_{3}\right)$, inequality (3.1) implies inequality (A.8), and estimate (A.10) implies estimate (3.3).

(b) We recall that estimate (A.4) is obtained by deriving estimates of $\|z\|_{2}$, $\|\nabla z\|_{2}$ and $\left\|\nabla^{2} z\right\|_{2}$, which is done by standard arguments involving the CauchySchwarz and Hölder inequalities. In particular, we get

$$
\begin{aligned}
\left(1-\|\nabla \boldsymbol{v}\|_{C^{0}}\right)\|z\|_{1,2} & \leq\|f\|_{1,2}, \\
\|z\|_{2,2} & \leq\|f\|_{2,2}+\alpha \theta\|\nabla z\|_{1,2},
\end{aligned}
$$

where $\alpha:=\max \left\{2, K_{6}\right\}$. Thus, if inequality (A.3) holds and $\boldsymbol{E}$ is the solution of equation (A.2), then $\|\nabla \boldsymbol{v}\|_{C^{0}}<1$ (since $\alpha>1$ ) and it follows from inequality (A.11) that $\|\boldsymbol{E}\|_{1,2} \leq\left(1-\|\nabla \boldsymbol{v}\|_{C^{0}}\right)^{-1}\|\boldsymbol{F}\|_{1,2}$. Thus, $L_{R}^{-1}$ is bounded in the topology of $H_{T}^{1}(\Omega)$ and

$$
\left\|L_{R}^{-1}\right\|_{1}^{*}:=\sup \left\{\left\|L_{R}^{-1} \boldsymbol{F}\right\|_{1,2}: \boldsymbol{F} \in H_{T}^{2}(\Omega),\|\boldsymbol{F}\|_{1,2}=1\right\} \leq\left(1-\|\nabla \boldsymbol{v}\|_{C^{0}}\right)^{-1} .
$$


Now suppose that inequality (3.1) holds and let $\boldsymbol{E}$ be the solution of equation (3.2). Then $\boldsymbol{E}=L_{R}^{-1}(\boldsymbol{F}-M \boldsymbol{E})$ and $\|\nabla \boldsymbol{v}\|_{C^{0}} \leq K_{0}\|\nabla \boldsymbol{v}\|_{2,2} \leq C_{2} \theta<1$ since $K_{0}<C_{2}$. Thus

$$
\|\boldsymbol{E}\|_{1,2} \leq\left(1-K_{0}\|\nabla \boldsymbol{v}\|_{2,2}\right)^{-1}\left(\|\boldsymbol{F}\|_{1,2}+\|M\|_{1}^{*}\|\boldsymbol{E}\|_{1,2}\right)
$$

and therefore $\left(1-\gamma_{1}\right)\|\boldsymbol{E}\|_{1,2} \leq\|\boldsymbol{F}\|_{1,2}$, where $\gamma_{1}$ is defined as in inequality (3.4) with $C_{1}:=K_{0}$. Hence, inequality (3.4) implies estimate (3.5).

(c) By definition of $L,(L \boldsymbol{E})^{T}=L\left(\boldsymbol{E}^{T}\right)$. If $(M \boldsymbol{E})^{T}=M\left(\boldsymbol{E}^{T}\right)$ then $(L+$ $M)\left(\boldsymbol{E}^{T}\right)=(L \boldsymbol{E}+M \boldsymbol{E})^{T}=\boldsymbol{F}^{T}=\boldsymbol{F}=(L+M) \boldsymbol{E}$ and thus $\boldsymbol{E}^{T}=\boldsymbol{E}$ because $L+M$ is injective. Alternatively, if $(M \boldsymbol{E})^{T}=M \boldsymbol{E}$ then $L\left(\boldsymbol{E}^{T}\right)=(L \boldsymbol{E})^{T}=$ $(\boldsymbol{F}-M \boldsymbol{E})^{T}=\boldsymbol{F}-M \boldsymbol{E}=L \boldsymbol{E}$ and thus $\boldsymbol{E}^{T}=\boldsymbol{E}$ because $L$ is injective.

\section{Acknowledgement}

I thank the reviewer for several comments which helped to improve this paper.

\section{References}

[1] J. G. Oldroyd, On the formulation of rheological equations of state, Proc. Roy. Soc. London Ser. A 200 (1950), 523-541.

[2] J. G. Oldroyd, Non-Newtonian effects in steady motion of some idealized elasticoviscous liquids, Proc. Roy. Soc. London Ser. A 245 (1958), 278-297.

[3] K. R. Rajagopal \& A. R. Srinivasa, A thermodynamic frame work for rate type fluid models, J. Non-Newtonian Fluid Mech. 88 (2000), 200-227.

[4] P. J. Oliveira, Alternative derivation of differential constitutive equations of the Oldroyd-B type, J. Non-Newtonian Fluid Mech. 160 (2009), 40-46.

[5] P. C. Bollada \& T. N. Phillips, On the mathematical modelling of a compressible viscoelastic fluid, Arch. Rational Mech. Anal. 205 (2012), 1-26.

[6] M. Renardy, Existence of slow steady flows of viscoelastic fluids with differential constitutive equations, Z. Angew. Math. Mech. 65 (1985), 449-451.

[7] C. Guillopé \& J. C. Saut, Existence results for the flow of viscoelastic fluids with a differential constitutive law, Nonlinear Anal. 15 (1990), 849-869.

[8] C. Guillopé \& J. C. Saut, Global existence and one-dimensional stability of shearing motions of viscoelastic fluids of Oldroyd type, M2AN Math. Model. Numer. Anal. 24 (1990), 369-401.

[9] C. Guillopé \& J. C. Saut, Existence and stability of steady flows of weakly viscoelastic fluids, Proc. Roy. Soc. Edinburgh 119A (1991), 137-158.

[10] C. Guillopé \& J. C. Saut, Mathematical problems arising in differential models for viscoelastic fluids, In: J. F. Rodrigues \& A. Sequeira (Editors), Mathematical topics in fluid mechanics, 64-92, Pitman Res. Notes Math. Ser. 274, Longman Scientific \& Technical, Harlow, Essex, 1992.

[11] E. Fernández-Cara, F. Guillén \& R. R. Ortega, Some theoretical results concerning non Newtonian fluids of the Oldroyd kind, Ann. Scuola Norm. Pisa Cl. Sci. (4) 26 (1998), 1-29.

[12] S. Matušů-Nečasová, A. Sequeira \& J. H. Videman, Existence of classical solutions for compressible viscoelastic fluids of Oldroyd type past an obstacle, Math. Meth. Appl. Sci. 22 (1999), 449-460.

[13] R. Talhouk, Existence results for steady flow of weakly compressible viscoelastic fluids with a differential constitutive law, Differential Integral Equations 12 (1999), 741-772. 
[14] M. A. Fontelos \& A. Friedman, Stationary non-Newtonian fluid flows in channel-like and pipe-like domains, Arch. Rational Mech. Anal. 151 (2000), 1-43.

[15] P. L. Lions \& N. Masmoudi, Global solutions for some Oldroyd models of nonNewtonian flows, Chin. Ann. Math. 21B (2000), 131-146.

[16] K. Pileckas, A. Sequeira \& J. H. Videman, Steady flows of viscoelastic fluids in domains with outlets to infinity, J. Math. Fluid Mech. 2 (2000), 185-218.

[17] S. D. Besbes \& C. Guillopé, Non-isothermal flows of viscoelastic incompressible fluids, Nonlinear Anal. 44 (2001), 919-942.

[18] M. A. Fontelos \& A. Friedman, Analysis of the stick-slip problem for non-Newtonian flows, Commun. Part. Diff. Eq. 26 (2001), 461-536.

[19] N. Arada \& A. Sequeira, Strong steady solutions for a generalized Oldroyd-B model with shear-dependent viscosity in a bounded domain, Math. Models Methods Appl. Sci. 13 (2003), 1303-1323.

[20] V. T. Dmitrienko, M. Kirane \& V. G. Zvyagin, On weak solutions for generalized Oldroyd model for laminar and turbulent flows of nonlinear viscous-elastic fluid, Nonlinear Anal. 53 (2003), 197-226.

[21] C. Guillopé \& R. Talhouk, Steady flows of slightly compressible viscoelastic fluids of Jeffreys' type around an obstacle, Differential Integral Equations 16 (2003), 12931320 .

[22] L. Chupin, Some theoretical results concerning diphasic viscoelastic flows of the Oldroyd kind, Adv. Differential Equations 9 (2004), 1039-1078.

[23] L. Molinet \& R. Talhouk, Existence and stability results for 3-D regular flows of viscoelastic fluids of White-Metzner type, Nonlinear Anal. 58 (2004), 813-833.

[24] L. Molinet \& R. Talhouk, On the global and periodic regular flows of viscoelastic fluids with a differential constitutive law, NoDEA Nonlinear Differential Equations Appl. 11 (2004), 349-359.

[25] D. A. Vorotnikov \& V. G. Zvyagin, On the solvability of the initial-value problem for the motion equations of nonlinear viscoelastic medium in the whole space, Nonlinear Anal. 58 (2004), 631-656.

[26] N. Arada \& A. Sequeira, Steady flows of shear-dependent Oldroyd-B fluids around an obstacle, J. Math. Fluid Mech. 7 (2005), 451-483.

[27] F.-H. Lin, C. Liu \& P. Zhang, On hydrodynamics of viscoelastic fluids, Comm. Pure Appl. Math. 58 (2005), 1437-1471.

[28] R. Salvi, Existence and uniqueness results for non-Newtonian fluids of the Oldroyd type, Int. Rev. Pure Appl. Math. 2005 (2005), 159-212.

[29] R. Salvi, Existence and uniqueness results for non-Newtonian fluids of the Oldroyd type in unbounded domains, In: Regularity and other aspects of the Navier-Stokes equations, 209-237, Banach Center Publ. 70, Polish Acad. Sci., Warsaw, 2005.

[30] S. Nečasová \& P. Penel, Incompressible non-Newtonian fluids: time asymptotic behaviour of solutions, Math. Meth. Appl. Sci. 29 (2006), 1615-1630.

[31] G. Bayada, L. Chupin \& S. Martin, Viscoelastic fluids in a thin domain, Quart. Appl. Math. 65 (2007), 625-651.

[32] A. Bonito, P. Clément \& M. Picasso, Mathematical and numerical analysis of a simplified time-dependent viscoelastic flow, Numer. Math. 107 (2007), 213-255.

[33] Q. Chen \& C. Miao, Global well-posedness of viscoelastic fluids of Oldroyd type in Besov spaces, Nonlinear Anal. 68 (2008), 1928-1939.

[34] Z. Lei, C. Liu \& Y. Zhou, Global solutions for incompressible viscoelastic fluids, Arch. Rational Mech. Anal. 188 (2008), 371-398. 
[35] F. Lin \& P. Zhang, On the initial-value problem of the incompressible viscoelastic fluid system, Comm. Pure Appl. Math. 61 (2008), 539-558.

[36] V. G. Zvyagin \& D. A. Vorotnikov, Approximating-topological methods in some problems of hydrodynamics, J. Fixed Point Theory Appl. 3 (2008), 23-49.

[37] G. Bayada, L. Chupin \& B. Grec, Viscoelastic fluids in thin domains: a mathematical proof, Asymptot. Anal. 64 (2009), 185-211.

[38] M. Renardy, Global existence of solutions for shear flow of certain viscoelastic fluids, J. Math. Fluid Mech. 11 (2009), 91-99.

[39] C. Guillopé, Z. Salloum \& R. Talhouk, Regular flows of weakly compressible viscoelastic fluids and the incompressible limit, Discrete Contin. Dyn. Syst. Ser. B 14 (2010), 1001-1028.

[40] L. He \& L. Xi, Global well-posedness for viscoelastic fluid system in bounded domains, SIAM J. Math. Anal. 42 (2010), 2610-2625.

[41] Z. Salloum, Flows of weakly compressible viscoelastic fluids through a regular bounded domain with inflow-outflow boundary conditions, Comm. Pure Appl. Anal. 9 (2010), 625-642.

[42] J. Qian \& Z. Zhang, Global well-posedness for compressible viscoelastic fluids near equilibrium, Arch. Rational Mech. Anal. 198 (2010), 835-868.

[43] T. Laadj \& M. Renardy, Initial value problems for creeping flow of Maxwell fluids, Nonlinear Anal. 74 (2011), 3614-3632.

[44] W. Zhao, The global existence of small solutions to the Oldroyd-B model, Chin. Ann. Math. 32B (2011), 215-222.

[45] P. Constantin \& M. Kliegl, Note on the global regularity for two-dimensional OldroydB fluids with diffusive stress, Arch. Rational Mech. Anal. 206 (2012), 725-740.

[46] M. Hieber, Y. Naito \& Y. Shibata, Global existence results for Oldroyd-B fluids in exterior domains, J. Differential Equations 252 (2012), 2617-2629.

[47] J. M. Marchal \& M. J. Crochet, A new mixed finite element for calculating viscoelastic flow, J. Non-Newtonian Fluid Mech. 26 (1987), 77-114.

[48] M. El Hadj, P. A. Tanguy \& A. Fortin, A numerical comparison of two decoupled methods for the simulation of viscoelastic fluid flows, Rheol. Acta 29 (1990), 342-351.

[49] R. G. Owens \& T. N. Phillips, A spectral domain decomposition method for the planar non-Newtonian stick-slip flow, J. Non-Newtonian Fluid Mech. 41 (1991), 43-79.

[50] J. Rosenberg \& R. Keunings, Numerical integration of differential viscoelastic models, J. Non-Newtonian Fluid Mech. 39 (1991), 269-290.

[51] R. I. Tanner \& X. Huang, Stress singularities in non-Newtonian stick-slip and edge flows, J. Non-Newtonian Fluid Mech. 50 (1993), 135-160.

[52] H. Al Moatassime \& C. Jouron, A Multigrid method for solving steady viscoelastic fluid flow, Comput. Methods Appl. Mech. Engrg. 190 (2001), 4061-4080.

[53] V. Ngamaramvaranggul \& M. F. Webster, Viscoelastic simulations of stick-slip and die-swell flows, Int. J. Numer. Meth. Fluids 36 (2001), 539-595.

[54] V. J. Ervin \& W. W. Miles, Approximation of time-dependent viscoelastic fluid flow: SUPG approximation, SIAM J. Numer. Anal. 41 (2003), 457-486.

[55] V. J. Ervin \& W. W. Miles, Approximation of time-dependent, multi-component, viscoelastic fluid flow, Comput. Methods Appl. Mech. Engrg. 194 (2005), 2229-2255.

[56] A. Liakos, Finite-element approximation of viscoelastic fluid flow with slip boundary condition, Comput. Math. Appl. 49 (2005), 281-294.

[57] M. Bensaada \& D. Esselaoui, Error estimates for a stabilized finite element method for the Oldroyd B model, J. Math. Anal. Appl. 325 (2007), 1042-1059. 
[58] S. Boyaval, T. Lelièvre \& C. Mangoubi, Free-energy-dissipative schemes for Oldroyd-B model, M2AN Math. Model. Numer. Anal. 43 (2009), 523-561.

[59] J. S. Howell, Computation of viscoelastic fluid flows using continuation methods, J. Comput. Appl. Math. 225 (2009), 187-201.

[60] J. C. Crispell, V. J. Ervin \& E. W. Jenkins, A fractional step $\theta$-method approximation of time-dependent viscoelastic fluid flow, J. Comput. Appl. Math. 232 (2009), 159-175.

[61] J. W. Barrett \& S. Boyaval, Existence and approximation of a (regularized) Oldroyd-B model, Math. Models Methods Appl. Sci. 21 (2011), 1783-1837.

[62] R. D. Wesson \& T. C. Papanastasiou, Flow singularity and slip velocity in plane extrudate swell computations, J. Non-Newtonian Fluid Mech. 26 (1988), 277-295.

[63] M. A. Spaid \& G. M. Homsy, Stability of Newtonian and viscoelastic dynamic contact lines, Phys. Fluids 8 (1996), 460-478.

[64] T. R. Salamon, D. E. Bornside, R. C. Armstrong \& R. A. Brown, Local similarity solutions for the stress field of an Oldroyd-B fluid in the partial-slip/slip flow, Phys. Fluids 9 (1997), 2191-2209.

[65] M. M. Denn, Extrusion instabilities and wall slip, Ann. Rev. Fluid. Mech. 33 (2001), 265-287.

[66] S. G. Hatzikiriakos \& K. B. Miglers (Editors), Polymer Processing Instabilities, Marcel Dekker, New York, 2005.

[67] J. M. Dealy \& R. G. Larson, Structure and Rheology of Molten Polymers, Carl Hanser Verlag, Munich, 2006.

[68] G. C. Georgiou, On the stability of the shear flow of a viscoelastic fluid with slip along the fixed wall, Rheol. Acta 35 (1996), 39-47.

[69] E. Brasseur, M. M. Fyrillas, G. C. Georgiou \& M. J. Crochet, The time-dependent extrudate-swell problem of an Oldroyd-B fluid with slip along the wall, J. Rheology 42 (1998), 549-566.

[70] M. M. Fyrillas \& G. C. Georgiou, Linear stability diagrams for the shear flow of an Oldroyd-B fluid with slip along the fixed wall, Rheol. Acta 37 (1998), 61-67.

[71] M. E. Kavousanakis, L. Russo, C. I. Siettos, A. G. Boudouvis \& G. C. Georgiou, A timestepper approach for the systematic bifurcation and stability analysis of polymer extrusion dynamics, J. Non-Newtonian Fluid Mech. 151 (2008), 59-68.

[72] C. Le Roux, Steady Stokes flows with threshold slip boundary conditions, Math. Models Methods Appl. Sci. 15 (2005), 1141-1168.

[73] R. A. Adams \& J. J. F. Fournier, Sobolev Spaces. 2nd Edition, Pure and Applied Mathematics 40, Academic Press, Amsterdam, 2003.

[74] H. Beirão da Veiga, Regularity for Stokes and generalized Stokes systems under nonhomogeneous slip-type boundary conditions, Adv. Differential Equations 9 (2004), 1079-1114.

[75] G. P. Galdi, An Introduction to the Mathematical Theory of the Navier-Stokes Equations. Volume 1: Linearized Steady Problems. Revised edition, Springer Tracts in Natural Philosophy 38, Springer Verlag, New York, 1994.

[76] S. Agmon, A. Douglis \& L. Nirenberg, Estimates near the boundary for solutions of elliptic partial differential equations satisfying general boundary conditions II, Comm. Pure Appl. Math. 17 (1964), 35-92.

[77] I. Hlaváček \& J. Nečas, On inequalities of Korn's type. II. Applications to linear elasticity, Arch. Rational Mech. Anal. 36 (1970), 312-337.

[78] J. Schauder, Der Fixpunktsatz in Funktionalräumen, Studia Math. 2 (1930), 171-180.

[79] C. Le Roux, Existence and uniqueness of the flow of second-grade fluids with slip boundary conditions, Arch. Rational Mech. Anal. 148 (1999), 309-356. 
[80] A. Tani \& C. Le Roux, Steady-state solutions to the equations of motion of secondgrade fluids with general Navier-type slip boundary conditions in Hölder spaces, Zap. Nauchn. Sem. S.-Peterburg. Otdel. Mat. Inst. Steklov. (POMI) 306 (2004), 210-228 (translation in J. Math. Sci. (N. Y.) 130 (2005), 4899-4909).

[81] C. Le Roux, On flows of third-grade fluids with non-linear slip boundary conditions, Int. J. Non-Linear Mech. 44 (2009), 31-41.

[82] H. Beirão da Veiga, On a stationary transport equation, Ann. Univ. Ferrara Sez. VII Sci. Mat. 32 (1986), 79-91.

[83] H. Beirão da Veiga, Existence results in Sobolev spaces for a stationary transport equation, Ricerche Mat. Suppl. 36 (1987), 173-184.

[84] A. Novotný, About steady transport equation I $-L^{p}$-approach in domains with smooth boundaries, Comment. Math. Univ. Carolinae 37 (1996), 43-89.

[85] A. Novotný, On the steady transport equation, In: J. Málek et al. (Eds.), Advanced topics in fluid mechanics, 118-146, Pitman Res. Notes Math. Ser. 392, Longman, Harlow, 1998.

Christiaan Le Roux

Department of Mathematics and Applied Mathematics

Faculty of Natural and Agricultural Sciences

University of Pretoria

Pretoria 0002

South Africa

e-mail: ianleroux@yahoo.com 